\title{
SYMPATHETIC OVERACTIVITY DUE TO LOCAL ANESTHETIC SYSTEMIC TOXICITY
}

\author{
Anna Spanomanoli, Stelios Michelis, Triantafyllia Dimou \\ Anesthesiology Department, Mediterraneo Hospital Athens, Greece
}

Background: Local anaesthetic systemic toxicity (LAST) typically appears $1-5$ minutes after injection. Initial manifestations vary widely. Classically, patients experience symptoms of central nervous system (CNS) excitation due to inhibition of inhibitory pathways. Soon CNS depression ensues. Aim: To present a case of LAST in a patient after a shoulder arthroscopy (SA).

Methods/Results: A 47-year-old female, diabetic, with good glycaemic control, ASA II, was scheduled for SA. General anaesthesia (GA) was administered, using midazolam for premedication, followed by propofol, fentanyl and rocuronium for induction and tracheal intubation. GA was maintained with desflurane and remifentanil infusion. The operation was uneventful and the patient after an uncomplicated recovery was transferred to the PACU.

The patient received paracetamol and pethidine and an interscalene brachial plexus block using a nerve stimulator was performed for postoperative analgesia. $15 \mathrm{mg}$ ropivacaine $0.5 \%$ were administered, using all safety measures (incremental injection $/$ careful aspiration) to avoid intravascular administration. 6 minutes later the patient was slightly confused and her blood pressure and heart rate progressively increased to $220 / 120 \mathrm{mmHg}$ and $160 \mathrm{bpm}$ respectively. Immediately midazolam, clonidine $150 \mu \mathrm{g}$ and esmolol infusion were used. Progressively haemodynamic status returned to normal and the patient returned to the ward after $60 \mathrm{~min}$ monitoring in the PACU.

Summary/ Conclusions: LAST may appear even when all safety measures are taken and doses kept minimum. Classical CNS symptoms as oral numbness, lightheadedness or seizures may be absent and sympathetic overactivity due to inhibition of inhibitory pathways of the sympathetic nervous system may be the cardinal clinical feature.

\section{Interscalene BP Block}

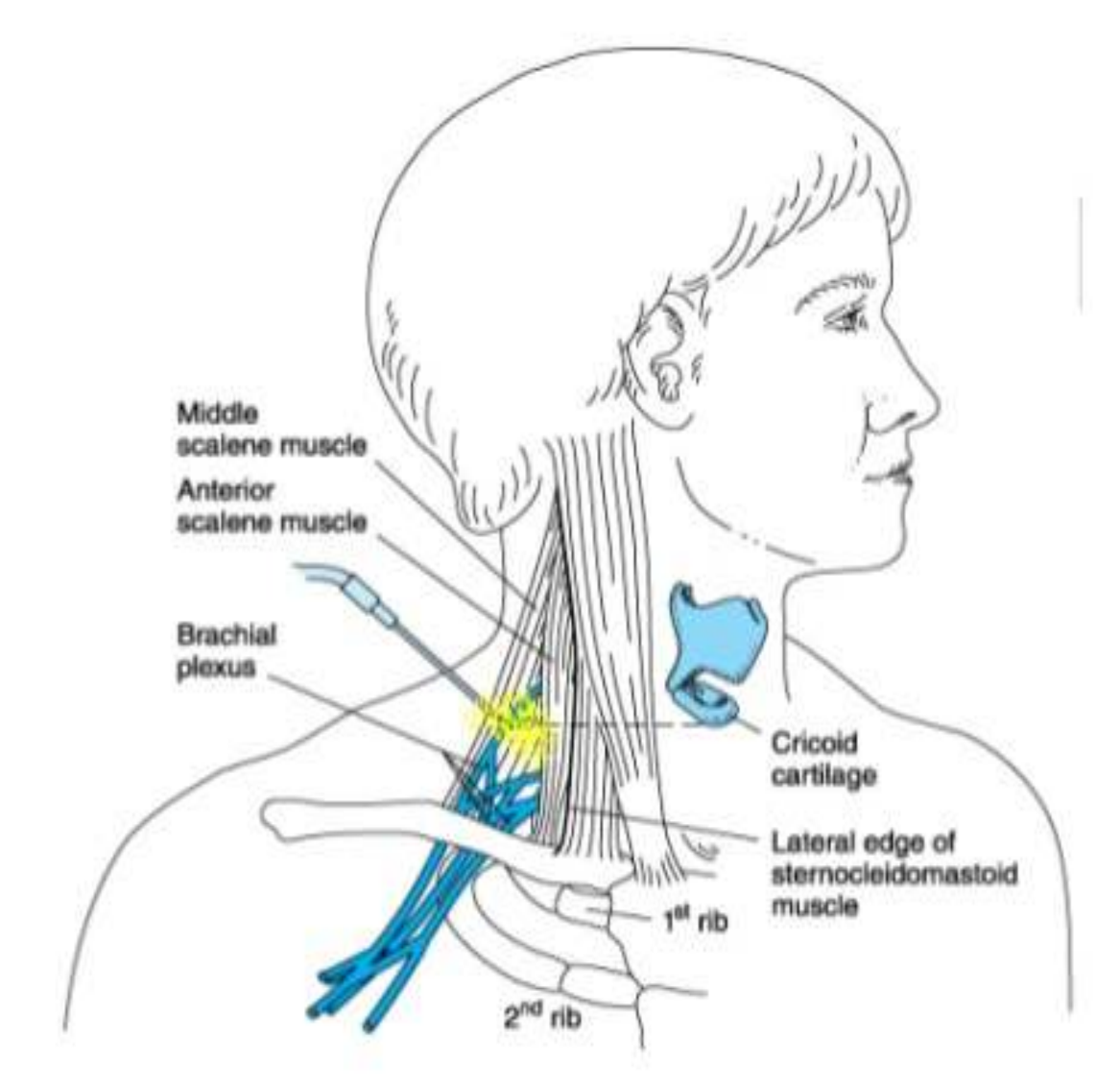

\section{DIAGNOSIS AND TREATMENT OF LOCAL ANESTHETIC TOXICITY}

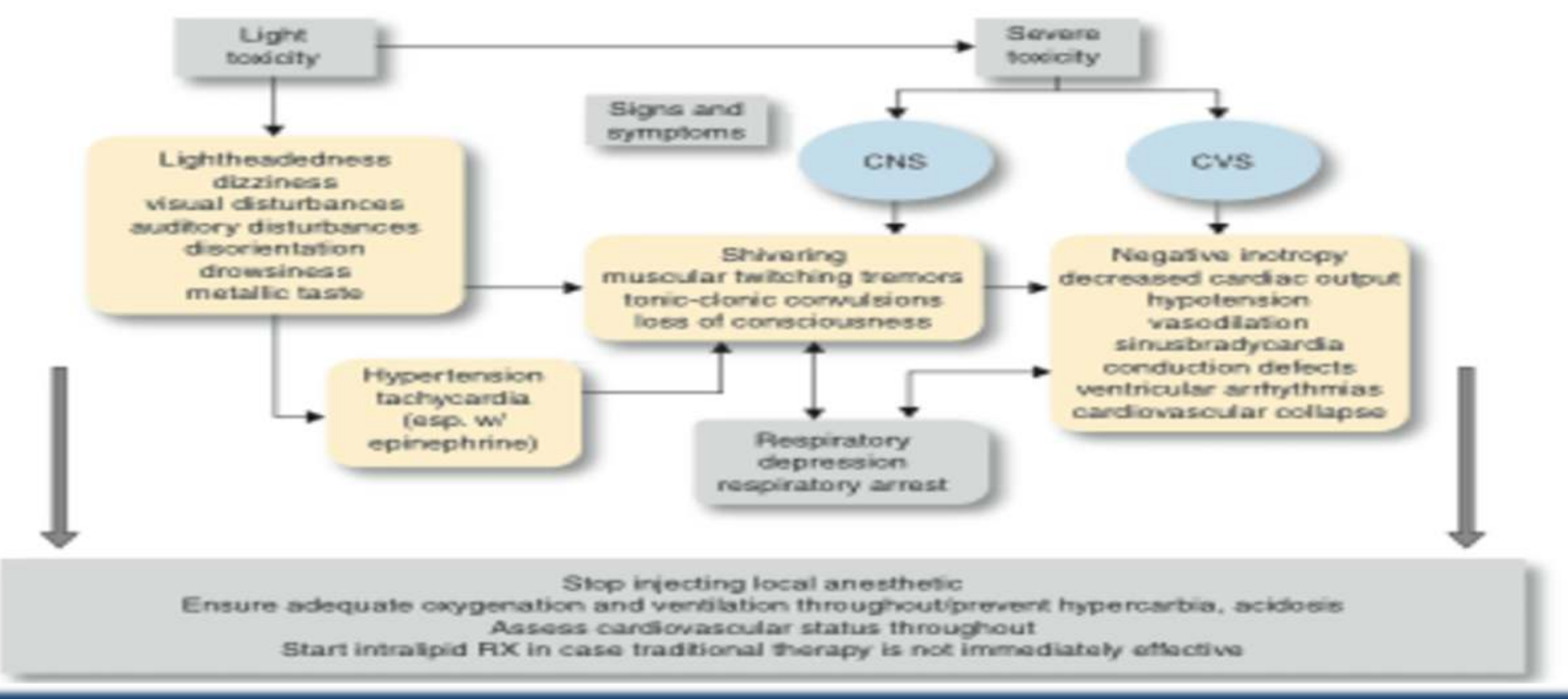

